INRA Prod. Anim.,

2014, 27 (3), 235-248

\title{
Le phénotypage de l'efficacité alimentaire et de ses composantes, une nécessité pour accroître l'efficience des productions animales
}

\author{
F. PHOCAS ${ }^{1,2}, J$. AGABRIEL ${ }^{3,4}$, M. DUPONT-NIVET ${ }^{1,2}$, I. GEURDEN ${ }^{5}$, F. MÉDALE ${ }^{5}$, \\ S. MIGNON-GRASTEAU ${ }^{6}$, H. GILBERT ${ }^{7,8,9}$, J.-Y. DOURMAD ${ }^{10,11}$ \\ ${ }^{1}$ INRA, UMR1313 GABI, F-78352 Jouy-en-Josas, France \\ 2 AgroParisTech, UMR1313 GABI, F-75231 Paris, France \\ 3 INRA UMR1213 Herbivores, F- 63122 Saint-Genès-Champanelle, France \\ ${ }^{4}$ Clermont Université, VetAgro Sup, UMR1213 Herbivores, BP 10448, F-63000 Clermont-Ferrand, France \\ ${ }^{5}$ INRA UR1067 Nutrition, Métabolisme et Aquaculture, Aquapôle, F-64310 St Pée-sur-Nivelle, France \\ ${ }^{6}$ INRA, UR83 Recherches Avicoles, F-37380 Nouzilly, France \\ 7 INRA, UMR1388 GenPhySE, F-3 31326 Castanet-Tolosan France \\ 8 Université de Toulouse INPT ENSAT, UMR1388 GenPhySE, F-31326 Castanet-Tolosan, France \\ ${ }^{9}$ Université de Toulouse INPT ENVT, UMR1388 GenPhySE, F-31076 Toulouse, France \\ 10 INRA, UMR1348 PEGASE, F-35590 Saint Gilles, France \\ 11 Agrocampus Ouest, UMR1348 PEGASE, F-35000 Rennes, France \\ Courriel :Florence.Phocas@jouy.inra.fr
}

L'élevage doit produire plus, mieux et à moindre coût afin de contribuer à assurer la sécurité alimentaire mondiale tout en préservant la planète. Pour rester compétitif face à la raréfaction croissante des ressources, le secteur européen des productions animales doit innover pour améliorer l'efficacité de l'utilisation des aliments par les animaux.

L'alimentation des animaux est l'un des postes les plus importants des coûts d'élevage, variant de 25 à $70 \%$ du coût total de production selon les filières et les systèmes de production (Bovin : Idele 2012, Poisson : CNA 2011, Porc : Ifip 2012, Volailles : Riffard et al 2011). D'après les organisations internationales comme la FAO ou la Banque Mondiale, la compétition pour les ressources alimentaires et l'usage des terres ne peut que s'accroître, en raison de la demande croissante en protéines animales au niveau mondial, du changement climatique global et de la production de biocarburants. Selon la FAO (2006), les productions animales produisent un tiers des protéines consommées par les hommes, en consommant un tiers des céréales produites et en utilisant $70 \%$ des terres agricoles de la planète. En outre, l'alimentation des animaux d'élevage affecte l'environnement. L'élevage utilise des ressources non renouvelables, en particulier d'énergie et de phosphore. Il produit aussi directement, sous forme de méthane $\left(\mathrm{CH}_{4}\right)$ et de protoxyde d'azote $\left(\mathrm{N}_{2} \mathrm{O}\right)$, environ $9 \%$ des émissions de Gaz à Effet de Serre (GES) issues des activités humaines (Gill et al 2010). L'emprein- te carbone des différentes filières est très variable ; par $\mathrm{kg}$ de viande produite, Gill et al (2010) estiment que les émissions de GES sont de $13 \mathrm{~kg}$ équivalent $\mathrm{CO}_{2}$ pour les viandes ovines et bovines et de 3 à $4 \mathrm{~kg}$ équivalent $\mathrm{CO}_{2}$ pour les viandes de volaille et de porc. Pour les ruminants, ces chiffres sont à relativiser du fait de la valorisation des surfaces en herbe, puits de carbone et non utilisables pour la production de céréales. Pour les monogastriques, malgré leur impact plus faible par kg de viande, la forte concentration des élevages dans certaines zones est source de problèmes environnementaux. L'élevage contribue également de manière significative aux phénomènes d'acidification, en relation avec les émissions d'ammoniac, et à l'eutrophisation, en relation avec l'enrichissement des eaux en nitrates et en phosphates. Au niveau français, l'élevage et les surfaces associées contribuent ainsi à environ $90 \%$ des émissions d'ammoniac, $70 \%$ des émissions de $\mathrm{N}_{2} \mathrm{O}$ et $50 \%$ des pertes de nitrates (Peyraud et al 2012).

Pour soutenir le développement durable des productions animales et tendre vers la notion d'élevage « écologique- ment intensif » (Griffon 2013), il est nécessaire de rechercher de nouvelles pratiques pour les systèmes de production et de nouveaux caractères au niveau des individus. Les nouvelles pratiques concernent en particulier le choix de ressources alimentaires permettant de limiter la compétition pour l'usage des terres et de réduire l'empreinte écologique des élevages notamment en optimisant la transformation des ressources alimentaires en produits. La production d'effluents (matières non digérées, déchets du métabolisme) est inversement liée à l'efficacité de transformation des aliments. Au niveau des individus, il faut donc rechercher des animaux plus efficaces, ingérant et valorisant mieux l'herbe pour les ruminants, acceptant des sources de protéines végétales (ou toutes autres alternatives) pour les poissons carnivores, acceptant des aliments de moindre qualité énergétique et protéique pour les monogastriques. Enfin, un déséquilibre dans la capacité des animaux à utiliser les différents nutriments (par exemple l'azote et le phosphore) conduit à des rejets dont la composition est également déséquilibrée par rapport aux besoins des plantes et à la législation relative à 
l'amendement des sols (Mignon-Grasteau et al 2010).

L'identification des leviers d'action pour améliorer l'efficacité de l'utilisation des aliments par les animaux passe par des mesures précises qui permettent de décomposer finement les processus d'ingestion et de digestion et les métabolismes énergétique, protéique et minéral dans des conditions d'élevage variées. Les enjeux du phénotypage autour de l'efficience de la transformation des aliments sont de piloter de manière plus individualisée en qualité et en quantité l'alimentation et de sélectionner des animaux plus robustes (Phocas et al 2014, ce numéro) et à haut niveau de production, tout en limitant d'une part, la consommation de ressources peu renouvelables et/ou directement valorisables en alimentation humaine et, d'autre part les émissions de gaz à effet de serre et d'ammoniac ainsi que les rejets d'azote et de phosphore dans les sols et les eaux. L'objet de cette synthèse est de préciser les questions scientifiques et les verrous techniques à lever en termes de phénotypage animal pour répondre à ces défis sociétaux dans les principales filières animales terrestres ou aquacoles.

\section{1 / Enjeux et verrous de connaissance scientifique}

L'Efficacité Alimentaire (EA) est un phénotype intégratif complexe qui fait intervenir plusieurs fonctions vitales de l'animal. L'EA peut être exprimée en termes d'efficacité de transformation de $\mathrm{kg}$ d'aliment en $\mathrm{kg}$ de produit, qu'il s'agisse de lait, d'œufs, ou de gain de poids pour les animaux producteurs de viande. C'est la définition donnée à l'EA dans l'ontologie ATOL (Le Bail et al 2014, ce numéro). Il serait préférable de parler d'efficacité d'utilisation de l'aliment pour signifier l'aptitude biologique de l'animal à transformer l'aliment ingéré en biomasse destinée à l'alimentation de l'Homme. La notion d'EA est plus compliquée à appréhender dans le cas des femelles qui doivent produire des jeunes sur plusieurs cycles de reproduction, en maintenant éventuellement leur croissance propre et en préservant leur capacité de reproduction.

Dans toutes les espèces, l'amélioration de l'efficacité alimentaire consiste à agir principalement sur l'utilisation et la partition des nutriments entre les fonctions d'entretien (à minimiser) et les fonctions de production (à maximiser).

Ces cinquante dernières années, les travaux sur l'EA ont essentiellement concerné l'animal en croissance ou en engraissement recevant des rations rela-

Figure 1. Décomposition fine des processus d'ingestion, de digestion et des métabolismes énergétique et protéique.

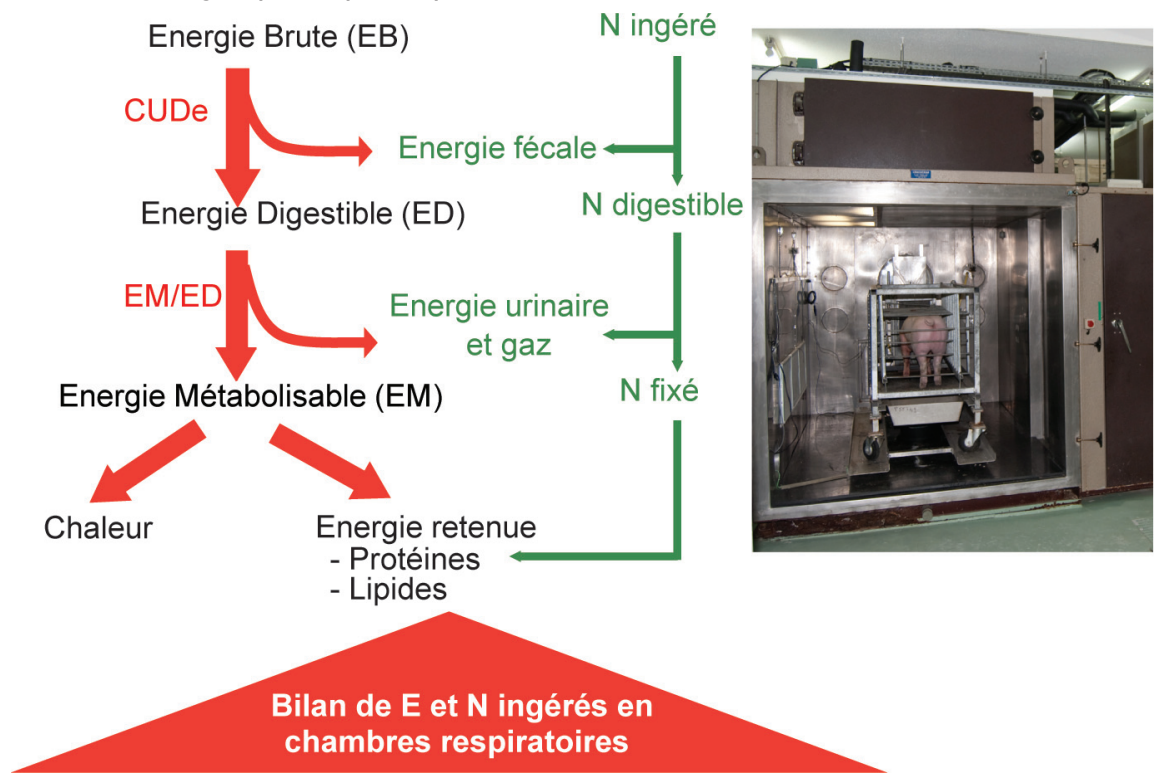

tivement riches en énergie. Cinq processus biologiques majeurs déterminent alors l'EA (Herd et Arthur 2009). Il s'agit de $i$ ) la capacité d'ingestion; ii) l'utilisation digestive de la ration; iii) l'efficacité métabolique (anabolisme et catabolisme, notamment associé aux variations de composition corporelle et de développement des viscères et organes digestifs); iv) la production de chaleur liée notamment à l'alimentation et l'activité physique et $v$ ) la thermorégulation chez les animaux homéothermes (figure 1). L'importance relative de ces différents processus est variable selon les espèces (figure 2 ) et les stades physiologiques (croissance, engraissement, gestation, lactation).

Les facteurs de variation de l'EA des femelles reproductrices sont moins bien connus que ceux de l'EA des animaux en croissance. A l'échelle du cycle de production d'une femelle reproductrice, d'autres facteurs de variation de l'EA sont à prendre en considération, notamment liés à la dynamique des réserves corporelles autour de la mise bas. La femelle mammifère stocke des lipides (énergie nette) avant de les mobiliser pour garantir la fin de la gestation et le début de la lactation. Ce processus plus ou moins marqué selon les espèces et leur niveau de sélection laitière se réalise avec des rendements variables modulant l'EA. Les déséquilibres de cette dynamique des réserves corporelles peuvent avoir des conséquences sur la santé et la fertilité ultérieure de la femelle (Berry et Crowley 2013). La longévité de la femelle reproductrice en est alors modifiée.

Chez les animaux en croissance, entre 50 et $70 \%$ des variations de l'ingéré s'expliquent par des différences dans la vitesse de croissance et le poids métabolique au sein d'un groupe d'animaux contemporains d'élevage (Bovins : Montanholi et al 2009, Kelly et al 2010 ; Porcs : Saintilan et al 2013 ; Volailles : de Verdal 2011 ; Poissons : Grima 2010). Il reste donc une variation individuelle considérable (environ 40\%) dans l'ingéré alimentaire autour de ce qui peut en être prédit à partir du poids et de la croissance des animaux dans toutes les espèces (Bovins : Archer et al 1999 ; Porcs : Gilbert et al 2007 ; Volailles : Luiting et Urff 1991 ; Poissons : Grima 2010). Les variations de cet ingéré résiduel (IR), correspondent :

- à des différences entre animaux dans les processus digestifs et métaboliques dont il s'agit de bien comprendre les mécanismes et les leviers d'action pour améliorer l'efficience de la production ;

- à des différences d'activité physique et de comportement;

- à des erreurs de mesure sur les quantités ingérées, le contenu du tube digestif, le poids et le gain de poids qui induisent une imprécision dans l'estimation des besoins des animaux (Robinson 2005).

Par ailleurs la composition des rations joue fortement sur l'EA. Il s'agit notamment de la nature du régime (densité énergétique, teneur en acides aminés par rapport aux besoins) et du niveau alimentaire alloué, avec des effets positifs ou négatifs selon les situations. Ainsi, dans beaucoup d'espèces, un rationnement modéré améliore l'EA, en particulier en limitant les dépôts de lipides. Les conditions environnementales comme la température et l'hygrométrie ont également un effet. Ainsi, l'animal homéotherme diminue son ingestion quand il fait chaud et humide et l'augmente quand il fait froid, alors que le poïkilotherme l'augmente jusqu'à la limite supérieure de ses 
Figure 2. Devenir de l'énergie ingérée chez trois types d'animaux d'élevage : a) la truite arc-en-ciel (Médale et Guillaume 1999), b) le taurillon à l'engraissement (Nkrumah et al 2006) et c) le porc en croissance (van Milgen et al 2008).

a. Truites arc-en-ciel élevées à $18^{\circ} \mathrm{C}$, poids $=150 \mathrm{~g}$ et $\mathrm{GMQ}=\mathbf{2 , 3} \mathrm{g} / \mathrm{j}$ consommant une ration de $2,3 \mathrm{~g} \mathrm{MS} / \mathrm{j}$ à $22 \mathrm{~kJ} / \mathrm{g} \mathrm{MS}$

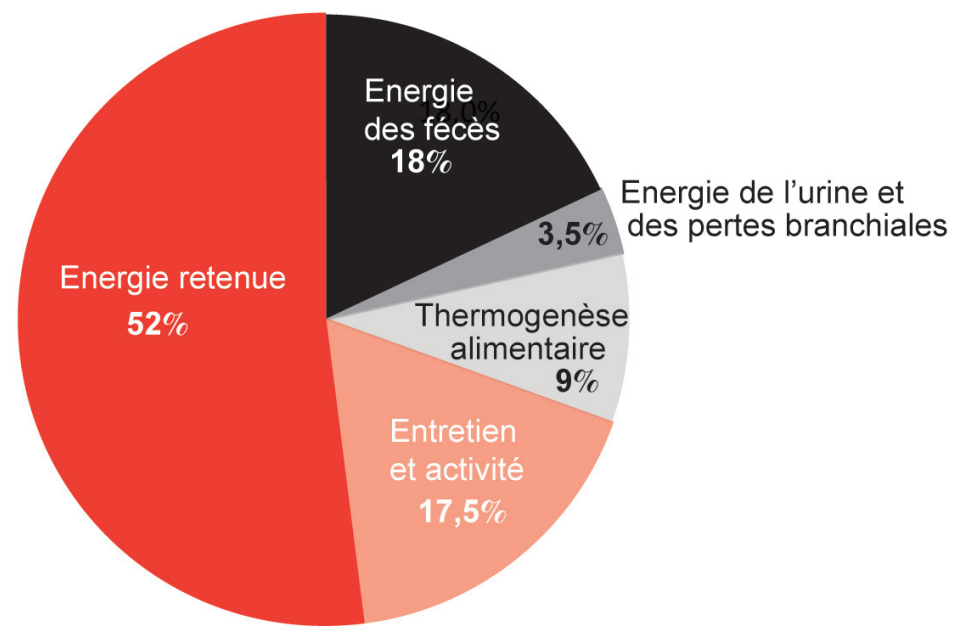

b. Taurillons de poids $=500 \mathrm{~kg}$ et $\mathrm{GMQ}=1,5 \mathrm{~kg} / \mathrm{j}$ consommant ad-libitum une ration de $11 \mathrm{~kg} \mathrm{MS} / \mathrm{j}$ à $19630 \mathrm{~kJ} / \mathrm{kg} \mathrm{MS}$

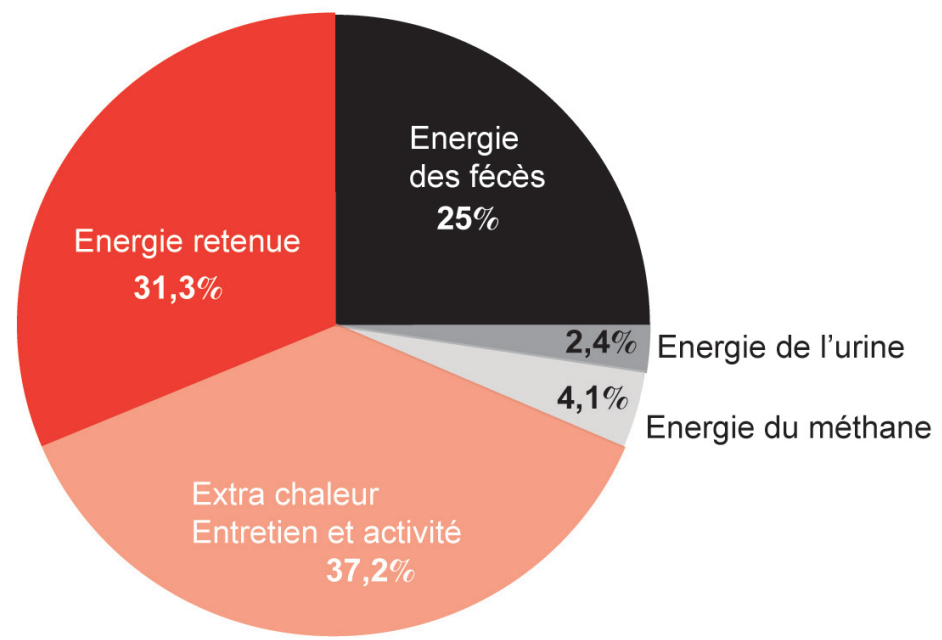

c. Porcs de poids $=65 \mathrm{~kg}$ et $\mathrm{GMQ}=0,9 \mathrm{~kg} / \mathrm{j}$ consommant ad-libitum une ration de $1,9 \mathrm{~kg} \mathrm{MS} / \mathrm{j}$ à $10000 \mathrm{~kJ} / \mathrm{kg} \mathrm{MS}$

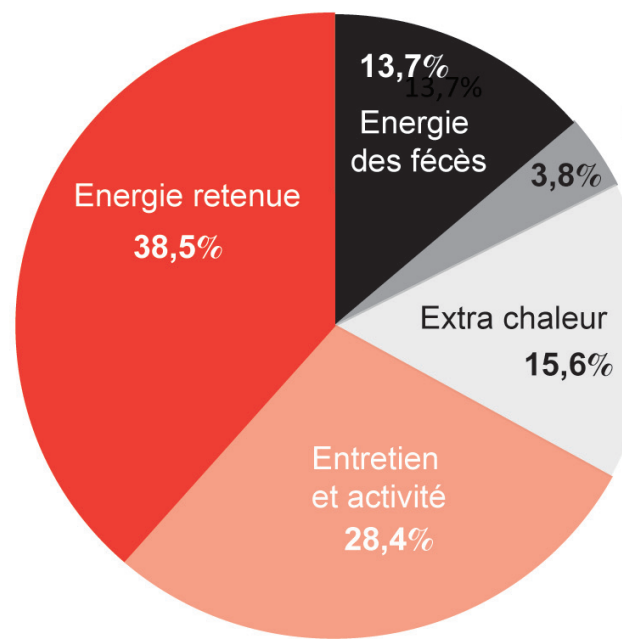

préférences thermiques (niveau au-delà duquel l'ingestion diminue alors fortement). En dernier lieu, la pression sanitaire peut aussi réduire l'EA. L'animal peut en effet utiliser une partie des nutriments pour la réponse immunitaire au lieu de la croissance.

Pour palier aux variations de concentration en énergie de l'aliment (MJ ingérés ou digestibles), l'EA est parfois exprimée en MJ d'énergie alimentaire / unité de produit (gain de poids, lait, œufs...). En effet, sous réserve que les acides aminés ou d'autres nutriments (comme les minéraux) ne soient pas limitants, le principal élément de la ration qui conditionne la production de l'animal est l'apport d'énergie nette (Noblet et al 1994b). Dans le cas contraire, c'est l'utilisation du nutriment limitant qui conditionne la réalisation des performances : par exemple, un régime dans lequel les acides aminés sont limitants conduit à un dépôt accru de lipides et moindre de protéines. Dans le contexte actuel, on a l'habitude de raisonner sur la base de l'énergie alimentaire, les autres facteurs étant supposés non limitants. Dans un souci de limiter les rejets azotés ou de minéraux, la question de l'efficacité d'utilisation des autres nutriments devient maintenant importante à considérer.

Afin d'explorer les voies possibles d'amélioration de l'EA, quatre questions de recherche majeures sont posées dans toutes les filières animales : $i$ ) le choix des critères de sélection de l'efficacité alimentaire ; ii) l'identification de la variabilité individuelle de l'efficacité digestive et du degré d'interaction entre le génotype et la valeur énergétique de la ration affectant cette efficacité digestive ; iii) l'étude de la variabilité individuelle de l'efficacité de l'utilisation des protéines et minéraux des aliments et $i v$ ) les conséquences des variations de l'EA sur l'empreinte environnementale des productions animales.

\section{1 / Quels critères de sélection de l'efficacité alimentaire?}

Dans la pratique, l'inverse de l'EA, c'est-à-dire le ratio entre quantité d'aliments ingérée et quantité de produit (viande, lait ou œufs), est souvent utilisé comme critère de mesure. Ce ratio est une mesure de l'efficacité de conversion des aliments en produits, appelé Indice de Consommation (cf. encadré).

Dans la littérature relative aux sciences des productions animales, plusieurs mesures sont également proposées sous l'appellation générique «efficacité alimentaire ». Outre l'IC, la mesure qui fait actuellement l'objet de beaucoup de recherches est l'IR (Ingéré Résiduel), aussi appelée consommation alimentaire 
Encadré. Relation entre deux critères de sélection de l'efficacité alimentaire : indice de consommation et ingéré résiduel.

L'Efficacité Alimentaire (EA) est l'inverse de l'Indice de Consommation (IC)

$E A=I C^{-1}=$ Quantité de produit $/$ Quantité d'aliment ingérée

L'Ingéré Résiduel (IR) est la différence entre l'ingéré réel (I_obs) et l'ingéré estimé (I_est).

Quantité d'aliment ingérée $=$ I_obs $=$ I_est + IR

I_est est l'ingéré estimé à partir des besoins nutritionnels d'entretien et de production (gain de poids, production laitière, poids d'œufs).

Les besoins d'entretien d'un animal au repos sont généralement estimés par une fonction linéaire du Poids Métabolique (PM) de l'animal, qui est le poids moyen calculé sur la période d'observation de l'EA et élevé à la puissance $p$ ( $p$ est généralement considéré égale à 0,75 et traduit le fait qu'un animal de faible poids a des besoins supérieurs en proportion qu'un animal de plus grande taille).

I_est $=\beta 0+\beta 1$ PM $+\beta 2$ Production $(+\beta 3$ Composition Corporelle $+\beta 4$ Activité $+\ldots)$

où les $\beta i$ sont les coefficients d'une régression linéaire multiple des variables explicatives de l'ingéré réel.

résiduelle (cf. encadré). L'IR représente l'écart entre l'ingéré réel d'un individu et son ingéré estimé sur la base de ses besoins nutritionnels d'entretien et de production (Koch et al 1963). Les besoins d'entretien correspondent aux dépenses minimales (à poids constant) pour le maintien de la vie au repos, liées aux grandes fonctions vitales telles que la respiration, la circulation sanguine, le renouvellement cellulaire, l'activité des muscles au repos etc. Les besoins de croissance et production couvrent à la fois la synthèse et l'accrétion des nutriments (protéines, lipides). Compte tenu des différences de teneur en eau et en énergie entre les tissus adipeux (très riches en énergie) et les tissus maigres (très riches en eau), ces besoins de production sont largement influencés par la composition du gain de poids (Robelin 1986, Noblet et al 1994a). Cependant, le modèle initial de Koch et al (1963) pour calculer l'IR ne prend pas en compte les différences d'efficacité énergétique entre dépôt de protéines et de lipides. L'efficacité énergétique du dépôt de lipides est environ 1,7 fois celle du dépôt de protéines, mais d'avantage d'eau (environ 4 fois plus) est retenue dans le processus de dépôt protéique que dans celui de dépôt du tissu adipeux (Owens et al 1995). En conséquence, les variations de l'efficacité relative des dépôts adipeux et protéiques est vraisemblablement un important facteur explicatif de l'IR, comme le suggèrent les travaux de Kelly et al (2010).

Des modèles de calculs de l'IR utilisent donc à présent des mesures de la composition corporelle (Basarab et al 2003, Saintilan et al 2013). Ce facteur est généralement retenu dans le calcul de l'IR quand la variabilité de composition corporelle est importante et mesurable entre animaux. Par exemple, chez la volaille ou le lapin, il n'est généralement pas utilisé (Drouilhet et al 2013). Pour beaucoup de productions animales, il est cependant important de disposer d'une mesure précise de la composition corporelle des animaux et de sa variation pour bien calculer le coût énergétique des dépôts lipidiques et protéiques. L'IR représente alors une EA nette des besoins de production. D'autres mesures d'efficacité ont été proposées (cf. synthèse de Berry et Crowley 2013), mais sont moins usitées à l'heure actuelle : elles visent à estimer un niveau de croissance attendue à ingéré donné (gain résiduel) plutôt qu'à estimer un niveau d'ingéré pour une croissance donnée, voire à combiner les deux concepts. Les réponses attendues sont très différentes en fonction de la stratégie de sélection considérée (Berry et Crowley 2013, Drouilhet et al 2013) : une sélection sur l'IR réduit l'ingéré à vitesse de croissance constante, alors qu'une sélection sur le gain résiduel augmente la vitesse de croissance à ingéré donné.

Sélectionner pour une augmentation (ou une diminution) d'un ratio (comme l'EA ou IC, respectivement) ne permet pas de maitriser a priori si la réponse passera par une variation du numérateur ou du dénominateur. Cela peut entraîner des réponses à la sélection indésirables. Il est donc préférable d'utiliser une autre mesure qui permette de raisonner à production constante, tel que l'IR. En effet, par construction, l'IR quantifie la quantité ingérée à niveau de production constant et permet donc de s'affranchir de la variation des coûts de production liée aux variations de niveau de performance dans l'estimation de l'EA. En cela, l'IR est la composante de l'EA indépendante du niveau de production des animaux, et se distingue complètement de l'EA en termes d'interprétation.
Un autre élément en faveur de l'IR comme moyen pour sélectionner des animaux sur l'efficacité alimentaire est que le niveau de production est généralement inclus dans l'objectif de sélection global d'une race ou d'une lignée pour obtenir une réponse économique élevée. Néanmoins, cette mesure reste globale, puisqu'elle n'est pas spécifique de mécanismes biologiques sous-jacents aux différences d'efficacité alimentaire entre animaux (efficacité digestive ou métabolique, par exemple). L'IR intègre en particulier des différences qui ne sont pas réellement des composantes de l'efficacité de transformation de l'aliment en produit, comme par exemple l'activité des animaux si celle-ci n'est pas prise en compte dans le calcul de l'ingéré estimé. Cependant, ce critère est soumis à une forte imprécision de mesure car il cumule toutes les erreurs faites sur chacun des termes inclus dans la prédiction de l'ingéré attendu des animaux. Berry et Crowley (2013) considèrent qu'une large part (environ 90\%) de la variabilité génétique de l'ingéré est expliquée tant chez des bovins en croissance qu'en lactation par cinq prédicteurs, à savoir le gain de poids, le poids, la composition corporelle, la morphologie et la production laitière, rendant une sélection de l'EA possible et efficace sans mesure de l'ingéré à large échelle chez les bovins.

Deux méthodes peuvent être utilisées pour estimer les besoins nutritionnels des animaux, base du calcul de l'ingéré théorique. La première consiste en un calcul du bilan énergétique. Elle s'appuie sur des modèles nutritionnels établis à partir des besoins d'entretien et de production. Ces modèles sont nombreux et les paramètres qui les composent sont très divers, par exemple la quantité d'énergie métabolisable contenue dans l'aliment, le coefficient d'efficacité d'utilisation de l'énergie métabolisable pour la production et l'entretien (calculé à partir de la densité énergétique du type de production) ou les quantités d'énergie requises pour l'accrétion de protéines et de lipides. La seconde méthode prédit les besoins de manière empirique à partir des données expérimentales en établissant la relation entre l'ingéré alimentaire et les caractéristiques de production de l'animal (gain de poids, poids d'œufs, production de lait...). C'est la méthode utilisée quand on fait un calcul d'IR par régression linéaire. Les quantités d'aliment requises pour les besoins d'entretien sont alors dépendantes du poids métabolique de l'individu. Ce dernier correspond au poids vif de l'individu affecté d'une puissance comprise généralement entre 0,60 et 0,80 , constante dérivée de la relation entre la dépense énergétique de l'animal à l'entretien et le poids. Les paramètres de la prédiction de l'ingéré varient donc d'une espèce à 
l'autre, mais aussi d'une étude à l'autre, en fonction des spécificités de production et des contraintes expérimentales (disponibilité des données, structure d'élevage, conditions environnementales...). L'IR estimé à partir des données propres à l'expérimentation étant plus facile à obtenir, il est plus souvent utilisé que l'IR estimé par les modèles nutritionnels.

La recherche de critères de sélection indirects ou bio-marqueurs de l'EA est un domaine important pour pouvoir prédire à moindre coût cette mesure difficile à réaliser en routine et à large échelle. Ainsi, les concentrations systémiques d'hormones jouant un rôle dans la partition et l'utilisation des nutriments, et des métabolites associés à la consommation alimentaire, la croissance, l'accumulation de graisse ou la répartition des éléments nutritifs ont attiré l'attention comme potentiels marqueurs physiologiques de l'EA. Chez le porc, des corrélations génétiques positives $(\mathrm{r}=0,63$ à 0,84$)$ ont été estimées entre EA et teneur en IGF1 mesurée entre 3 et 10 jours post sevrage, mais d'autres métabolites mesurés dans le sang ou les urines ne sont généralement pas liés fortement avec l'EA (Lefaucheur et al 2011). Chez les bovins allaitants (Richardson et al 2004, Moore et al 2005, Montanholi et al 2010, Kelly et al 2010), des corrélations phénotypiques et génétiques ont été déterminées entre IR et plusieurs métabolites dosés dans le sang (glucose, insuline, créatinine, aspartate, aminotransférase, cortisol, leptine, IGF-1), l'urine et les fèces. Globalement, les corrélations entre ces potentiels bio-marqueurs et les mesures de consommation, performance et EA, restent souvent faibles et parfois non significativement différentes de zéro. Les corrélations établies sont notamment dépendantes du stade physiologique auquel ces bio-marqueurs sont mesurés. Ceci souligne la nécessité d'explorer d'autres types de bio-marqueurs.

\section{2 / Variabilité individuelle de l'efficacité digestive et interaction avec la valeur énergétique de la ration}

L'amélioration de l'EA a été réalisée essentiellement dans un contexte où l'accès à des ressources alimentaires « nobles » n'était pas limitant : la sélection s'est effectuée sur des animaux alimentés avec des rations peu fibreuses, riches en énergie, faciles à digérer. La première question qui se pose donc en termes d'amélioration de l'EA à moindre coût économique et environnemental est de savoir si les animaux les plus efficaces pour des rations riches en énergie sont aussi les animaux les plus efficaces pour des rations riches en fibres, peu digestibles pour les monogastriques?
Chez les monogastriques, la sélection pour une meilleure EA a essentiellement amélioré l'efficacité métabolique globale, principalement en modifiant la nature des dépôts (moins gras) et en diminuant la part de l'entretien (croissance ou production plus élevée), mais elle a très peu affecté l'efficacité digestive. En effet, l'utilisation de régimes à base de matières premières de haute qualité énergétique sollicite peu les capacités digestives des animaux. Chez les animaux en croissance ou en engraissement, l'efficacité de la transformation de l'énergie digestible en énergie métabolisable est très élevée (91\% chez le bovin, Nkrumah et al 2006 ; $96 \%$ chez le porc, Noblet et al 1994b). En revanche l'efficacité de la transformation de l'énergie brute en énergie digestible est nettement moindre $(75 \%$ chez le bovin, Nkrumah et al $2006 ; 81 \%$ chez le porc, Noblet et al 1994b), mais variable selon la nature de l'énergie brute des aliments. Ceci suggère un levier d'action potentiellement important pour réduire les rejets (notamment d'origine fécale) : l'amélioration de l'efficacité digestive des animaux. Quelque soit l'espèce considérée, il existe très peu de travaux mesurant la variabilité individuelle de la digestibilité de l'énergie et des nutriments. Chez les bovins, Dulphy et al (1995) considèrent que cette variabilité est faible avec un coefficient de variation sur un lot d'animaux de $2 \%$, mais cela représente près de 6 points de digestibilité d'écart entre l'animal qui digère le plus et celui qui digère le moins. Cette variabilité pourrait être plus spécifiquement ciblée pour permettre une meilleure valorisation de ressources alimentaires diversifiées par les animaux d'élevage. Les travaux pionniers de Mignon-Grasteau et al (2004) chez le poulet et de Noblet et al (2013) chez le porc ont ainsi montré l'existence d'une base génétique exploitable pour améliorer l'efficacité digestive de l'énergie et des nutriments de la ration. Dans ces études, le phénotype est typiquement soumis à une interaction de type génotype $\times$ aliment, et il n'est révélé qu'en condition de challenge alimentaire. Ainsi, si les animaux ayant une bonne aptitude à digérer des aliments difficiles à digérer se révèlent capables de bien digérer des aliments faciles à digérer, l'inverse n'est pas vrai. Une compréhension approfondie des facteurs génétiques et d'élevage contrôlant l'adaptabilité à la variabilité de la qualité des aliments (liés à l'animal et/ou à son microbiote) est ainsi nécessaire pour définir les meilleures stratégies de sélection et d'alimentation des animaux.

\section{3 / Variabilité individuelle de l'efficacité de l'utilisation pro- téique et minérale des aliments}

Une troisième question essentielle pour toutes les espèces dans les années à venir concerne l'efficacité de l'utilisa- tion protéique et minérale des aliments. Comme indiqué précédemment, l'essentiel des travaux sur l'efficacité alimentaire ont été conduits en supposant que les apports en acides aminés et en minéraux ne limitent pas la couverture des besoins d'entretien et de production des animaux. Or, dans le but d'accroître l'autonomie européenne dans l'approvisionnement en protéines végétales, ainsi que pour limiter les rejets azotés dans l'environnement, il paraît nécessaire d'explorer les possibilités d'amélioration de l'efficacité protéique. Toutefois très peu de résultats sont disponibles à ce sujet dans la bibliographie, même si certains laissent présager une variabilité entre animaux à la fois en termes d'efficacité digestive et métabolique (Volailles : de Verdal et al 2011 ; Poissons : Glencross et al 2007). Ainsi chez la vache laitière où les apports en énergie et protéines interagissent sur les performances de production, Brun-Lafleur et al (2010) montrent que ces interactions sont très dépendantes du potentiel de production de la vache. Dans toutes les espèces, on ne sait pas à l'heure actuelle expliquer précisément pourquoi un animal est plus efficace qu'un autre pour déposer des protéines. Cette efficacité variable de la transformation des protéines alimentaires induit également des rejets d'azote dans l'environnement très variables entre animaux (van Milgen et al 2008), même si le premier facteur à maîtriser en termes de limitation des rejets est évidemment l'adéquation entre les apports et les besoins nutritionnels des animaux.

\section{4 / Amélioration de l'empreinte environnementale des productions animales}

Les animaux présentant l'efficacité alimentaire la plus élevée sont aussi ceux qui rejettent le moins d'effluents par unité de produit (Bovins : Nkrumah et al 2006 ; Poissons : Kaushik 1990 ; Porcs : Dourmad et Jondreville 2007 ; Volailles : de Verdal et al 2013). Se pose alors une question importante au moins pour les filières terrestres : peut-on directement réduire la quantité de rejets qu'ils soient solides, liquides ou gazeux ? En termes d'émission de gaz à effet de serre, cela concerne essentiellement le protoxyde d'azote provenant des effluents pour toutes les filières, et le méthane entérique pour les ruminants (Hegarty et al 2007) et dans une moindre mesure pour le porc. En termes d'effluents, l'amélioration de la composition des rejets, par exemple en améliorant l'équilibre du ratio azote/ phosphore des rejets des monogastriques (Mignon-Grasteau et al 2010) ou encore en les adaptant en fonction de leur utilisation ultérieure, comme par exemple la production d'énergie par méthanisation (Jarret et al 2012), est aussi à envisager. 


\section{2 / Enjeux et verrous de connaissance technique}

La levée des verrous scientifiques présentés ci-dessus nécessite des mesures précises pour décomposer finement les processus d'ingestion, de digestion et les métabolismes énergétique et protéique dans des conditions d'élevage variées, ceci avant d'identifier les leviers d'action pour améliorer l'efficacité de l'utilisation des aliments.

\section{1 / La mesure des quantités ingérées}

Dans toutes les filières, il est difficile de mesurer de manière fiable une composante essentielle de l'efficacité alimentaire : les quantités d'aliment ingérées par chaque animal. En plus d'être précise, une bonne mesure doit être enregistrée en perturbant le moins possible les comportements sociaux et de compétition dans les groupes d'animaux pour l'accès à la nourriture, quelque soit la situation d'élevage : la difficulté est particulièrement prononcée chez les poissons en milieu aquatique (Silverstein et al 2005), mais aussi chez les volailles au sol et les ruminants au pâturage.

Un pilotage de précision de l'alimentation, stratégie d'avenir pour adapter la ration délivrée aux besoins journaliers de chaque animal, nécessite l'acquisition de connaissances sur chaque individu via des mesures individuelles, et non plus un pilotage de l'alimentation par lot. Alors que le pilotage individuel est techniquement possible en bovins et porcins (Pomar et al 2014), son déploiement reste à mettre en œuvre chez les volailles (Basso et al 2010) et il est difficilement envisageable chez les poissons (Kause et al 2002, Kolstad et al 2004).

Cette mesure des quantités ingérées, même quand elle est réalisable, reste coûteuse à obtenir et est généralement limitée à un sous échantillon d'animaux. Pour une utilisation à large échelle, il s'agit de trouver des mesures indirectes de ce phénotype. Chez les poissons, on cherche ainsi un critère de tri permettant d'élever des lots très homogènes en termes de besoins alimentaires pour réduire la variabilité individuelle intra-lot. Chez les animaux terrestres, les approches basées sur les mesures automatisées à haute fréquence sur l'animal vivant, telles que l'imagerie pour la note d'état corporel, les accéléromètres ou les vidéos pour le comportement alimentaire et l'ingestion, la thermographie, les sondes ruminales etc., sont prometteuses, mais restent à développer dans un contexte de recherche d'efficacité alimentaire. La valeur de l'utilisation conjointe de ces technologies pour l'estimation de l'ingestion et des composantes des besoins des animaux n'a pas encore été décrite. C'est un nouveau champ à explorer.

\section{2 / Les cinétiques de croissance, d'ingestion et de composition corporelle}

L'accès à des poids et des quantités ingérées en cours de croissance permet de décrire des cinétiques de croissance et d'ingestion, notamment grâce à des modèles pour le porc (InraPorc $\AA$ ) ou le bovin (Hoch et Agabriel 2004). Ces cinétiques permettent de décomposer l'efficacité alimentaire en fonction des périodes de la croissance, et d'extrapoler les besoins en nutriments des animaux à différentes périodes, éventuellement au niveau individuel. Elles mettent par exemple en évidence que les animaux les plus efficaces ont des besoins nutritionnels supérieurs aux autres animaux pendant la croissance avec la nécessité d'un apport supérieur en acides aminés. Il apparaît aussi que des animaux dont la vitesse de croissance est identique pendant la période d'élevage peuvent avoir une meilleure efficacité alimentaire en ayant une vitesse de croissance plus lente au démarrage, mais qui persiste plus longtemps (Saintilan 2012). Paradoxalement ces données sont relativement faciles à obtenir pour la dimension « ingestion » sous réserve que l'on dispose d'automates d'enregistrement, mais plus difficiles à collecter pour la dimension « poids » puisque l'automatisation des pesées est encore rare en élevage, même expérimental.

Comme indiqué précédemment, l'efficacité d'utilisation de l'aliment varie avec la composition de l'aliment ainsi qu'avec la composition des tissus déposés. La composition corporelle varie notamment avec le poids, l'âge, le sexe, le stade physiologique et le potentiel génétique des individus. La prédiction de la composition corporelle à partir d'équations a une précision variable selon les espèces, les types génétiques et les conditions d'élevage. Toutefois, ces dernières années, des techniques échographiques ou d'imagerie se sont développées pour valider les prédictions. Elles mesurent des épaisseurs de dépôts adipeux ou de muscles. Leur précision est parfois limitée par la nature très maigre des animaux d'élevage modernes, mais elle s'améliore avec les nouveaux appareils disponibles.

L'enregistrement objectif, systématique et non invasif de l'évolution de la composition corporelle, qui permettrait d'affiner encore les modèles, n'est donc pas encore développé, principalement pour des raisons de coût et de facilité de mise en œuvre. En outre, des cinétiques fines des poids et des notes d'appréciation manuelle et visuelle d'état corporel semblent être dans certains cas d'une fiabilité suffisante pour apprécier le bilan énergétique de l'animal (Thorup et al 2012). Les méthodes basées sur les échographies à ultra-sons en 2D et 3D sont encore peu développées en France. La technique échographique en $2 \mathrm{D}$ a été testée et validée dans plusieurs pays, depuis une dizaine d'années sur vaches allaitantes (Reverter et al 2000; Guideline BIF, $8^{\text {eme }}$ édition, 2002), et plus récemment aussi sur vaches laitières (Schroder et Staufenbiel 2006). L'imagerie 3D pourrait donner en outre une description volumétrique plus précise, intéressante pour la description dynamique de l'évolution de l'état corporel et de nombreux indicateurs morphologiques de l'animal (Bewley et al 2008, Halachmi et al 2008). Chez le porc, une première estimation de la composition corporelle peut être obtenue par la mesure par échographie de l'épaisseur de gras dorsal. Cette mesure est réalisée depuis de nombreuses années pour la sélection et plus récemment pour le pilotage de l'alimentation des truies, mais requiert l'intervention humaine. La qualité des images obtenues par les échographes actuels permet également d'évaluer l'épaisseur du muscle long dorsal voire son persillé chez les types génétiques les plus gras. D'autres méthodes, comme la tomographie par scanner à rayon $\mathrm{X}$, permettent une évaluation précise de la composition corporelle des porcs et des volailles, mais elles nécessitent dans la plupart des cas une anesthésie préalable garante de l'immobilité des animaux.

\section{3 / La mesure des rejets et des émissions}

La détermination des rejets et des émissions renseigne sur l'efficacité d'utilisation des nutriments et sur certains processus biologiques sous-jacents. Elle est en outre importante pour la maîtrise de l'utilisation des effluents, la connaissance de leur composition étant indispensable pour leur bonne valorisation comme fertilisant ou pour la production d'énergie. Cependant, indépendamment de la méthode de mesure de l'excrétion se pose la question de la signification biologique des critères mesurés. En effet une excrétion élevée peut résulter de processus biologiques (digestion, métabolisme) peu efficaces ou d'un apport excessif des nutriments (protéines, phosphore) ou encore d'un déséquilibre de l'apport alimentaire en acides aminés par rapport aux besoins des animaux. La notion d'excrétion est donc très liée aux conditions d'élevage des animaux.

La mesure directe des effluents des animaux nécessite la collecte totale des excrétas sur des périodes de plusieurs jours. Différents dispositifs expérimen- 
taux existent pour réaliser ces mesures, y compris chez les poissons. Ce sont les mêmes équipements que ceux utilisés pour établir des bilans nutritionnels, par exemple pour déterminer la digestibilité des nutriments et de l'énergie ou leur rétention par les animaux (Demarquilly et al 1995 pour les ruminants). Notons en particulier l'apport déterminant de la spectrométrie dans le proche infra-rouge, en rendant possible l'estimation rapide de la composition chimique des fèces (Bastianelli et al 2010), voire directement de la digestibilité. La collecte séparée de l'urine et des fèces permet d'avoir des renseignements sur l'origine des excrétas, c'est-à-dire de déterminer s'ils proviennent de la fraction apparemment indigestible de l'aliment ou s'ils ont une origine métabolique. Cette répartition dépend de la composition de l'aliment, mais aussi du fonctionnement du rumen ou du gros intestin qui sont en partie caractéristiques de l'individu et/ou de la flore qu'il héberge. Toutefois, comme la séparation entre fèces et urine n'est pas possible chez les volailles, il faut alors recourir à l'utilisation d'un marqueur de la fraction solide. L'utilisation de marqueurs indigestibles permet d'estimer l'excrétion fécale sur la base d'un échantillon de fèces et de la concentration relative du marqueur dans la ration et dans les excreta.

Ces mesures sont généralement réalisées sur des animaux logés en cages ou stalles individuelles ou sur des groupes de poissons élevés dans des bassins équipés d'un système de récupération automatiques des fèces. Des manipulations immédiates sont indispensables pour stabiliser les échantillons, par exemple acidifier et/ou refroidir l'urine pour éviter les émissions d'ammoniac, ce qui rend le phénotypage complexe. L'estimation de la composition des effluents en élevage constitue aussi un enjeu important pour un pilotage de précision de leur utilisation. Des dispositifs existent permettant de collecter les effluents en mélange pour des animaux élevés en groupe, mais on obtient alors une valeur moyenne et non une valeur individuelle. Compte tenu des émissions gazeuses, en particulier d'ammoniac, ces dispositifs conduisent d'ailleurs à une sous-estimation de l'excrétion.

Une autre méthode pour estimer les rejets est de les calculer par différence entre l'ingestion d'une part, et la rétention et la production d'autre part. Les productions (lait, œufs, nouveau-nés) sont généralement faciles à mesurer chez tous les animaux, à l'exception des animaux allaitants pour lesquels la production de lait doit être estimée (Saintilan et al 2013). Pour les animaux en croissance, la rétention corporelle peut être calculée à partir de modèles prenant en compte le poids des animaux et des indicateurs de composition corporelle. On rejoint alors la problématique du phénotypage de la composition corporelle évoquée cidessus, avec dans le cas de l'excrétion, la nécessité de phénotyper également la rétention minérale.

La mesure des émissions gazeuses des animaux revêt une importance particulière en raison de l'influence des gaz émis sur l'environnement, en particulier le méthane. Les mesures des émissions de $\mathrm{CO}_{2}$ et de $\mathrm{CH}_{4}$ et de la consommation $\mathrm{d}^{\prime} \mathrm{O}_{2}$ font partie des mesures de routine pour les études se rapportant au métabolisme énergétique. Elles sont généralement réalisées dans des chambres respiratoires qui permettent de déterminer précisément les flux d'air et la composition de l'air entrant et sortant. Il s'agit de méthodes très couteuses qu'il est difficile d'envisager en routine sur de grands effectifs. Aussi ces dernières années, des méthodes alternatives basées sur l'utilisation de marqueurs ont été développées pour évaluer la production de méthane entérique chez les ruminants Muñoz et al 2012). Récemment, l'utilisation d'analyseurs de gaz à l'échelle de l'élevage a permis de mettre en évidence des différences d'émissions gazeuses de groupes de volailles à faible ou forte efficacité digestive élevés dans la même salle (Mignon-Grasteau et al 2013). Ces méthodes nécessitent encore des adaptations avant de pouvoir être utilisées pour le phénotypage individuel de ces émissions.

L'utilisation de marqueurs biologiques, en particulier de certains composés du lait, a également été proposée pour estimer les rejets ou les émissions. Sous certaines conditions, l'excrétion azotée

Figure 3. Système d'alimentation à la demande chez les poissons (dessin d'A. Gélineau).

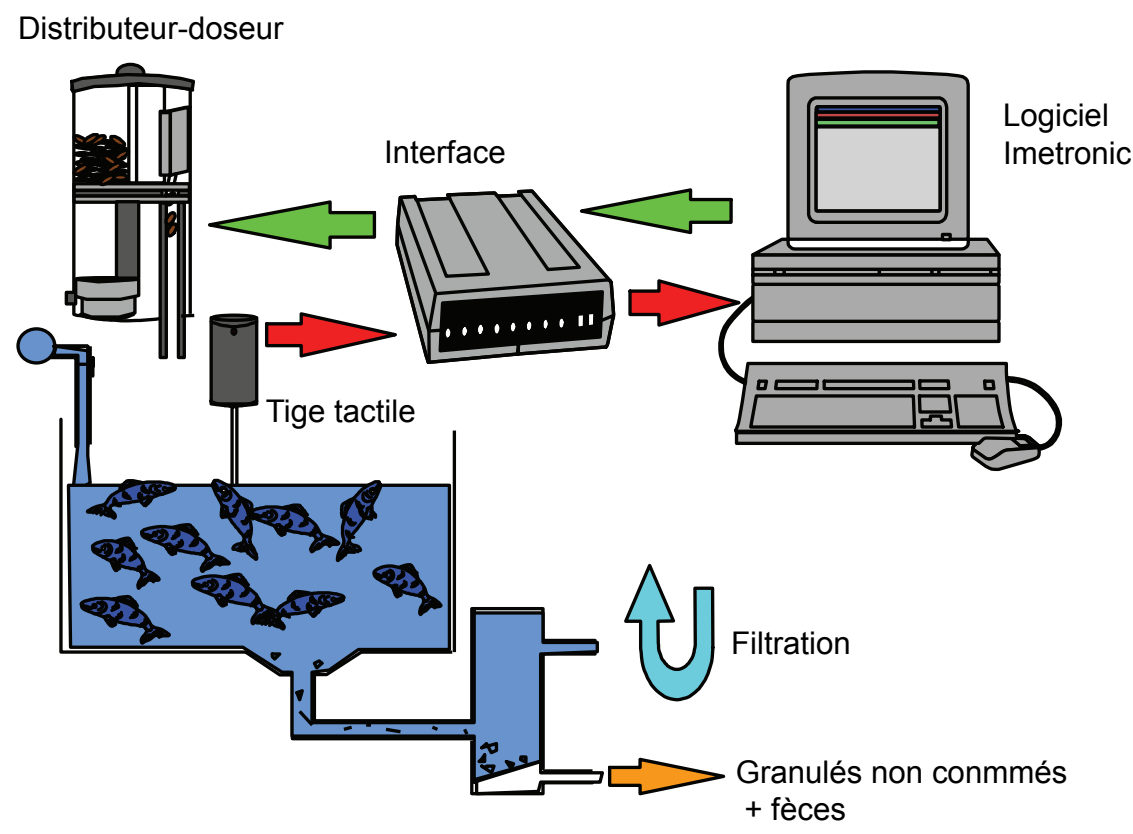

dans le lait pourrait ainsi permettre d'estimer l'excrétion azotée des vaches laitières. De même, la composition en acides gras du lait qui est influencée par le métabolisme du rumen pourrait permettre de prédire la production de méthane entérique (Dehareng et al 2012). Toutefois ces méthodes restent à affiner.

\section{3 / Défis spécifiques pour l'amélioration de l'efficacité alimentaire}

\section{1 / Les défis spécifiques aux animaux aquatiques}

En aquaculture, le défi majeur reste la quantification de l'ingéré. En effet, l'aliment est distribué aux poissons soit manuellement à satiété visuelle, soit par un distributeur automatique (figure 3), Même pour ces deux dernières méthodes d'alimentation, il est nécessaire de quantifier l'aliment non-consommé afin d'optimiser l'estimation des quantités ingérées. Ceci peut se réaliser à l'aide d'un tamis placé à la sortie de chaque bassin, ce qui nécessite la séparation des granulés non consommés des fèces, les deux subissant un fort taux de lessivage (méthode lourde et contraignante). Une autre méthode consiste à restreindre les quantités d'aliment distribuées, par exemple à 1,5\% de la biomasse du poisson, pendant toute la période de mesure de l'efficacité alimentaire.

Chez le poisson, l'ingéré est le plus souvent mesuré au niveau du groupe, mais ceci impose de travailler avec des familles génétiques élevées séparément soit « à la demande » (Boujard et al 1992). 
(Kolstad et al 2004), avec les contraintes connues de ce type de dispositif : réplications des bassins d'élevage, coûts élevés en installations expérimentales et inadéquation aux systèmes d'élevage des PME françaises impliquées dans la sélection. L'alternative est la mesure de l'ingéré individuel. Elle peut être obtenue en élevant les poissons en compartiment individuel (Silverstein et al 2005), mais cela reste extrêmement coûteux et il n'a pas été démontré que le comportement des poissons isolés soit représentatif de leur comportement en groupe. Une autre possibilité est l'utilisation des rayons $\mathrm{X}$ : des billes radio-opaques sont incluses dans l'aliment, et les poissons sont radiographiés après leur repas (Kause et al 2002). Cette méthode est cependant lourde et délicate. En particulier, les mesures d'ingéré doivent être répétées un grand nombre de fois par individu, pour tenir compte des importantes variations journalières d'ingéré alimentaire à l'échelle individuelle (Kause et al 2002, Grima et al 2008). Or, les manipulations répétées engendrent un stress qui peut perturber l'appétit, nuisant à la représentativité des mesures dans les dispositifs de sélection.

Des travaux ont été conduits récemment à l'INRA (Grima et al 2008 chez la truite, Grima et al 2010 chez le bar) pour rechercher des critères indirects individuels, fortement corrélés à l'efficacité alimentaire. Deux critères ont été testés : la perte de poids pendant le jeûne et la reprise de poids pendant la croissance compensatrice après le jeûne. La perte de poids pendant le jeûne est un indicateur des besoins d'entretien (une forte perte durant le jeûne correspond à des besoins d'entretien élevés et une efficacité alimentaire plus faible). La reprise de poids pendant la croissance compensatrice après le jeûne est prise comme un indicateur d'efficacité métabolique de rétention des nutriments. Il a été montré chez la truite commune que la reprise de poids est associée à des variations de l'ingéré résiduel (Mambrini et al 2004).

L'autre défi majeur pour la production aquacole est l'optimisation de l'efficacité protéique dans le contexte de substitution de la farine de poisson, considérée comme source idéale pour couvrir les besoins des poissons. Les poissons sont d'excellents transformateurs des protéines alimentaires. Carnivores, ils nécessitent des rations plus concentrées en protéines (32 à 55\% de la ration selon les espèces) que les autres animaux d'élevage. Ils sont donc particulièrement sensibles à la qualité de l'apport protéique alimentaire, et tout déséquilibre par rapport aux besoins entraine une baisse du taux de rétention et des rejets azotés accrus. Des recherches doivent être développées pour trouver des bio-marqueurs (métaboliques ou moléculaires) pertinents de l'efficacité d'utilisation protéique, aisément mesurables à large échelle. Des résultats récents montrent des modifications de la flore intestinale des poissons en fonction des sources protéiques du régime (Desai et al 2012) ; cependant, les relations entre flore intestinale et efficacité d'utilisation protéique restent à explorer.

\section{2 / Les défis spécifiques à l'avi- culture}

Chez les volailles, la mesure individuelle de l'ingéré est difficile à obtenir dans les conditions réelles d'élevage. Des initiatives ont d'ores et déjà été développées par certains sélectionneurs, mais sont le plus souvent limitées à la dernière phase de l'élevage. A l'INRA, des dispositifs ont été développés chez le canard (Basso et al 2010) ou sont en cours de développement chez le poulet (INRA 2013), afin de mesurer l'ingéré sur l'intégralité de la période d'élevage, en minimisant les facteurs pouvant perturber la consommation alimentaire.

Compte tenu de la difficulté encore forte de l'accès à l'information individuelle sur l'ingéré dans les conditions réelles d'élevage, les autres voies d'amélioration ont été privilégiées en aviculture. La minimisation des besoins d'entretien a été réalisée par l'augmentation de la vitesse de croissance, qui réduit de manière conséquente la durée d'élevage (environ 35 jours à l'heure actuelle contre 42 jours dans les années 1990). De plus, la forme de la courbe de croissance a également été affectée, avec une croissance très rapide sur la fin de la période d'élevage, ce qui diminue les besoins d'entretien en début d'élevage. La sélection de poulets de chair à croissance rapide a également abouti à une diminution considérable de l'activité physique des animaux. Un poulet de chair passe ainsi près de $90 \%$ de son temps couché, contre seulement $50 \%$ pour un poulet label (Bizeray et al 2000). Les différentes expériences de sélection montrent également que selon le critère de sélection de l'efficacité alimentaire des animaux, ce ne sont pas les mêmes composantes de l'efficacité qui sont affectées. La sélection de l'IR a peu affecté l'étape digestive, mais essentiellement l'étape métabolique post-digestive (composition corporelle, production de chaleur). La sélection de poules pondeuses sur l'IR a ainsi fortement affecté la production de chaleur des animaux (Bordas et al 1992, Gabarrou et al 1997). A l'opposé, la sélection de l'efficacité digestive a peu affecté l'étape post-digestive, les animaux sélectionnés sur l'énergie métabolisable corrigée pour un bilan azoté nul ne pré- sentant pas de différence majeure de composition corporelle, de température ou d'activité physique (de Verdal et al 2013).

Les poulets de chair sont de gros consommateurs de céréales (maïs, blé) et de soja. Il reste à évaluer la sensibilité de ces génotypes au remplacement d'une partie de ces matières premières par d'autres n'entrant pas en concurrence avec l'alimentation humaine (notamment les co-produits de la culture des céréales destinées à l'alimentation humaine) ou par des ressources protéiques plus locales. Les poulets de chair à croissance rapide qui sont fortement sélectionnés sur la vitesse de croissance et l'efficacité alimentaire ont cependant besoin, pour exprimer leur potentiel génétique, de régimes comprenant des matières premières énergétiques et protéiques de très haute qualité. La marge de manœuvre pour ces génotypes est donc probablement moindre que pour des productions alternatives (poulets certifiés à croissance intermédiaire, poulets labels à croissance lente par exemple).

Il est également intéressant de travailler sur l'efficacité de l'animal à transformer les différentes composantes de l'aliment (énergie, azote, phosphore), en liaison avec les rejets. En effet, par rapport à la législation actuelle, les rejets des volailles sont proportionnellement trop riches en phosphore, ce qui impliquerait de limiter la quantité de rejets épandus et d'ajouter des engrais azotés. Or, entre l'excrétion et l'épandage, le CORPEN (2006) a estimé que près de $50 \%$ de l'azote excrété était perdu et de Verdal et al (2011) ont montré que selon la capacité des animaux à digérer leur aliment, cette proportion devrait être limitée à une valeur comprise entre 15 et $35 \%$. C'est donc en combinant stratégies nutritionnelles, génétiques et de gestion de l'élevage qu'un progrès significatif pourra être obtenu.

Enfin, récemment, des travaux chez les volailles ont également montré l'importance du microbiote sur l'efficacité digestive. La composition du microbiote, particulièrement l'équilibre entre certains types de bactéries du tube digestif, affecterait l'efficacité digestive pour les composantes énergétiques, lipidiques, protéiques et pour l'amidon. L'existence d'un contrôle génétique de l'hôte semble également probable (Gabriel et al 2012). Des études couplées de métagénomique et de métabolomique semblent nécessaires pour prendre en compte l'ensemble du microbiote et ses interactions avec l'hôte, car différents types de bactéries peuvent avoir des rôles équivalents dans le tube digestif, et une simple étude de la composition du microbiote ne permettra pas d'aller jusqu'aux conséquences fonction- 
nelles de l'impact du microbiote sur l'efficacité alimentaire.

\section{3 / Les défis spécifiques à la filière porcine}

Chez le porc, le phénotypage de l'efficacité alimentaire passe, comme dans les autres espèces, par le phénotypage des quantités ingérées et du gain de poids, voire pour plus de précision, de la composition du gain de poids. Parmi ces caractères, le phénotypage individuel précis de l'ingestion est le plus délicat à mettre en œuvre dans des conditions d'élevage classiques en groupe, mais plusieurs dispositifs commerciaux existent. Le phénotypage du gain de poids est rarement automatisé, mais facilement réalisé à l'aide de balances en élevage. Les développements technologiques des 20 dernières années ont abouti à la mise au point d'automates d'alimentation des porcs avec identification électronique, qui permettent l'enregistrement de l'ingestion en conditions relativement proches des conditions d'élevage classiques en groupe. Ces automates sont cependant loin d'être présents en routine dans les élevages de sélection ou les stations de contrôle de performance, en raison de leur coût élevé. Les limites majeures des modèles commerciaux sont :

- l'absence de possibilité de rationnement des animaux ; ceci constitue une limite en situation d'élevage de production dans la mesure où les animaux sont souvent rationnés, mais n'en est pas une en sélection puisque cette dernière est réalisée en régime ad libitum ;

- une surexpression de la compétition entre animaux, qui se traduit par une détérioration des performances globales, sauf à avoir plusieurs automates dans chaque loge ;

- l'absence de souplesse dans la composition des aliments délivrés individuellement ; typiquement, il est difficile d'ajuster la composition de l'aliment délivré aux besoins spécifiques de chaque animal du groupe, ce qui est incompatible avec la notion d'alimentation de précision. Toutefois certains dispositifs expérimentaux commencent à le permettre (Pomar et al 2014).

Le phénotypage de l'EA chez les porcs concerne traditionnellement les porcs en croissance entre 25 et $110 \mathrm{~kg}$ de poids vif, c'est-à-dire entre 10 à 27 semaines d'âge environ. La période de post-sevrage, entre 3-4 et 10 semaines d'âge, est généralement ignorée, alors qu'elle constitue une période critique pour la santé digestive, l'animal passant au sevrage d'un aliment lacté à un aliment solide avec plus ou moins de facilité. A l'heure actuelle, les dispositifs d'enregistrement fiables et automatisés de l'ingestion n'existent pas pour cette période d'éleva- ge particulièrement sensible, en dehors de prototypes expérimentaux.

En termes d'évaluation de l'efficacité digestive des porcs se posent deux challenges majeurs pour le phénotypage : i) les différences d'efficacité digestive individuelles ne semblent révélées qu'avec des régimes qui « challengent » l'appareil digestif, par exemple des régimes plus fibreux et ii) la mesure individuelle de la capacité digestive n'est actuellement possible que chez des porcs élevés en cages individuelles, avec une collecte journalière des excrétas. Il faut donc d'une part, se donner les moyens de contrôler les animaux élevés avec des gammes d'aliments variées, et d'autre part, mettre au point un système de mesure de la digestibilité simple et précis, utilisable en élevage ou en station de contrôle. On pourrait s'inspirer des techniques développées chez les volailles qui sont en avance sur ce point (Mignon-Grasteau et al 2004). L'association entre efficacité alimentaire, composition et fonctionnalités du microbiote, en lien avec la capacité digestive des animaux, est aussi une question émergente. La question du phénotypage à haut débit des populations microbiennes grâce à la métagénomique est alors posée.

Une partie de la variation d'efficacité alimentaire est liée à des variations de besoins pour l'entretien et l'activité des animaux. Les variations de l'activité peuvent être traitées pour partie au travers du phénotypage automatisé de l'ingestion, car l'alimentation est une part majeure de l'activité des porcs et les automates d'alimentation enregistrent tous les évènements alimentaires (temps passé, nombre de visites...) pour chaque individu. Un phénotypage plus fin de l'activité physique à l'aide de caméras ou d'accéléromètres par exemple, permet de mesurer plus précisément encore ces différences. Il a par exemple été montré sur des lignées de porcs divergentes pour l'IR que la différence d'activité entre lignées expliquait $14 \%$ de la différence d'énergie ingérée (Meunier-Salaün et al 2013). Cependant, l'automatisation des enregistrements vidéo et de leurs analyses n'est pas triviale, et les données récoltées au niveau des automates sont largement sous-exploitées. Le développement d'algorithmes permettant de les traduire en termes d'activité, ou de relations de compétition au sein du groupe d'animaux est donc nécessaire pour améliorer le phénotypage de l'activité physique.

\section{4 / Les défis spécifiques à la filière bovine}

Chez les bovins allaitants comme laitiers, la capacité à ingérer puis transformer efficacement des fourrages, et à prélever de l'herbe pâturée quelles que soient sa qualité et sa nature sont des qualités recherchées à tout âge de la carrière. Il s'agit de développer:

- l'analyse de l'aptitude à prélever et à valoriser au mieux les ressources herbagères en considérant séparément les fourrages pâturés et conservés. Il faut obtenir des mesures directes ou indirectes du comportement et des choix alimentaires, et des mesures automatisées d'ingestion et de digestion. Le nombre de sites équipés pour ce type de mesures est extrêmement limité. Les mesures de comportement et d'ingestion alimentaires au pâturage nécessitent un système complexe d'enregistrement combinant des capteurs sensibles selon divers axes, GPS et caméra sur chaque animal. L'herbe offerte doit être mieux caractérisée (densité et homogénéité du couvert, hauteur d'herbe, composition botanique, valeur nutritive). L'ingestion d'herbe peut s'apprécier par la dilution de marqueurs indigestibles (Delagarde 2001) soit de type terres rares (ytterbium) soit de type alcanes (Mayes et al 1986). Chacune de ces techniques s'adaptent plus ou moins bien selon le type de prairie naturelle ou temporaire (Lawrence et al 2011 et 2012). La collecte totale des fèces et la concentration des marqueurs dans les fèces permettent alors l'estimation indirecte de la digestibilité de la matière organique. Une alternative à la mesure par marqueur est la mesure infra-rouge des fèces en reliant le spectre obtenu à l'ingestibilité et la digestibilité de l'herbe (Decruyenaere $e t$ al 2009). Sa validation est en cours d'essai à l'INRA y compris sur pâturage tropical en Guadeloupe ;

- la modélisation de l'efficience des vaches et de sa variabilité face à des aléas nutritionnels. Il s'agit de construire les trajectoires de croissance et d'évolution des tissus corporels des vaches de la naissance à l'âge adulte (Martin et Sauvant 2010). En races allaitantes, il faut développer pour cela des estimateurs de l'aptitude des dépôts lipidiques à se constituer lors des périodes d'alimentation favorable ou au contraire à être mobilisés avec un rendement élevé en période de disette. En races laitières, la mobilisation des réserves corporelles est essentielle quand l'ingéré est insuffisant pour couvrir les dépenses de lactation. C'est le cas notamment pour les vaches laitières hautes productrices en début de lactation. L'équilibre à rechercher entre le degré de mobilisation des réserves permettant une bonne production laitière et ses conséquences négatives sur la reproduction, la santé et la longévité des vaches est différent selon l'efficacité alimentaire des animaux. Celle-ci doit donc être mesurée sur des races de potentiels très différents placés dans des milieux variés ;

- une meilleure estimation des besoins d'entretien. Actuellement le seul estimateur est la mesure du niveau énergétique 
apporté pour une variation nulle du poids vif par la méthode du bilan complet. Une estimation indirecte est possible par la mesure du rendement de carcasse d'un individu, indiquant l'importance des viscères (poids relatif), et qui pourrait être corrélé avec ce besoin. La mesure automatisée de la thermographie infrarouge au niveau de diverses parties du corps, en particulier des pattes et de la joue, semble une voie possible aussi pour expliquer des pertes thermiques variées et comprendre ainsi indirectement les variations d'IR (Montanholi et al 2009).

A niveau de production donné, la variabilité de la production de méthane est génétiquement déterminée. Elle pourrait être négativement corrélée à l'efficacité alimentaire (Hegarty et al 2007) car la production de méthane correspond à une perte d'énergie pour l'animal. Ceci a été clairement montré chez des bovins en phase d'engraissement, mais les résultats sur vaches adultes sont peu nombreux et moins clairs. Une étude a montré que des vaches Angus sélectionnées sur un IR faible en postsevrage produisent moins de méthane quand elles pâturent une herbe de haute qualité, mais aucune différence n'est observée avec des vaches à haut IR quand la qualité du pâturage est dégradée (Jones et al 2011). Deux approches sont généralement utilisées pour mesurer cette production de méthane, soit en utilisant un gaz traceur (SF6) délivré à un rythme constant par un bolus ruminal, soit en mesurant par moyen infrarouge (MIR) les concentrations en méthane et $\mathrm{CO}_{2}$ dans une simili-chambre respiratoire, qui peut être adaptée au robot de traite ou au distributeur d'alimentation des animaux (par exemple le « Greenfeed system » (C-lock-in) testé à l'INRA de Bourges).

Dans le futur, la métagénomique devrait aussi fournir une estimation de la composition de la flore ruminale et de son interaction avec l'hôte, et à terme permettre de prédire l'efficacité du processus de fermentation (Morgavi et al 2013).

\section{4 / Perspectives d'amélioration de l'efficacité alimentaire}

La première limite à une amélioration rapide de l'efficacité alimentaire à l'échelle des populations animales concerne donc la mesure individuelle des quantités ingérées. Si des dispositifs de mesure de la consommation individuelle pour des animaux élevés en groupe ont été ou sont en cours d'élaboration dans toutes les filières, il reste néanmoins à affiner leur développement et diminuer leur coût afin de les déployer largement. Les pro- ductions de monogastriques bénéficient d'un contrôle de l'ingéré depuis quinze ans qui a permis de renforcer la pression de sélection directe sur l'EA (de Verdal 2011, Saintilan 2012) en combinaison avec la forte amélioration indirectement réalisée par l'augmentation de la vitesse de croissance et la diminution de l'adiposité des carcasses depuis plus de cinquante ans. Une sélection efficace et directe n'est pas encore possible à large échelle chez les ruminants (Bouquet et al 2010) et chez les poissons (Grima 2010). Il s'agit de réfléchir à des systèmes d'information à deux étages. D'une part, des populations de référence, utiles notamment pour la sélection génomique ou pour établir des équations de prédiction phénotypique doivent être mises en place. Dans ce cas, des mesures précises de l'EA et de ses composantes sont effectuées. Pour des raisons de coût, ces populations de référence ne peuvent se constituer à l'échelle nationale uniquement et devront s'étendre à l'international afin d'accroître les effectifs et ainsi permettre des prédictions précises. De grands projets internationaux voient ainsi le jour chez les ruminants. Chez les monogastriques, la structure en entreprises privées concurrentes ne facilite pas la mise en commun de données pour l'établissement d'une population de référence. D'autre part, des populations commerciales d'animaux, où des critères indirects de l'EA seraient mesurés, permettraient de contribuer à un pilotage de précision de l'alimentation. Dans toutes les espèces et filières, il y a un besoin important d'un phénotypage expérimental fin des animaux pour comprendre les facteurs de variations de l'EA et de ses composantes et identifier clairement les leviers d'action, ou proposer des équations de prédiction de l'ingéré et de l'EA basées sur des mesures plus faciles à déployer à large échelle (croissance, bio-marqueurs...).

L'efficacité alimentaire est un phénotype complexe (efficacité digestive / efficacité métabolique ; efficacité énergétique / efficacité protéique) qui est très sensible aux niveaux d'apports alimentaires et à la composition de la ration (énergie, acides aminés, digestibilité des fourrages). Pouvoir phénotyper les animaux dans certaines situations d'élevage standardisées et contrastées pour évaluer la variabilité de leur potentiel pour l'EA permettrait de faire un bond significatif dans le travail prospectif sur l'efficacité alimentaire. L'ambition d'obtenir en routine des évaluations type normes de réaction, c'est-à-dire des réponses à des variations d'environnement continues (Knap et Su 2008), est globalement irréaliste en élevage, en raison de la quantité d'enregistrements nécessaires à l'estimation précise de ces normes de réaction. En revanche, il semble réaliste de tester en élevages expérimentaux ou dans des dispositifs de contrôle de performance, des combinaisons d'aliments et de génotypes différents en nombre limité. La composition de l'aliment et son impact sur l'efficacité digestive individuelle reste dans ce cadre le poste qui recouvre le plus d'inconnues à ce jour. Chez les monogastriques, dans un contexte où la corrélation entre performances en élevages de sélection et performances en production est loin d'atteindre 1, la question de la robustesse des animaux sélectionnés à un environnement dégradé (statut sanitaire) ou même différent (ambiance) est également essentielle.

En termes d'efficience globale des productions animales, il s'agit enfin d'apprécier le lien avec les rejets en quantité (GES, N, P) et en qualité (rapport N/P par exemple) et les interrelations entre variations de l'efficacité alimentaire et activité ou santé. Les lignées divergentes créées à l'INRA chez la poule pondeuse (Bordas et al 1992) et le porc (Gilbert et al 2007) en utilisant la consommation résiduelle comme critère de sélection de l'efficacité alimentaire, offrent des possibilités particulièrement intéressantes pour étudier les relations entre l'efficacité alimentaire et les autres fonctions vitales, car elles présentent des divergences importantes en termes d'utilisation des nutriments et en particulier de l'énergie. Chez le poulet de chair, des lignées divergentes existent pour la capacité digestive (Mignon-Grasteau et al 2004); elles sont particulièrement intéressantes pour explorer la composante digestive de l'efficacité alimentaire, qui a été peu étudiée jusqu'à présent. Pour les poissons, les lignées isogéniques de truites créées par l'INRA constituent aussi un matériel de choix pour étudier les composantes de l'EA dans la mesure où certaines lignées s'avèrent plus performantes que d'autres pour un ingéré identique. Enfin, en plus de l'aliment et de l'animal, un troisième acteur doit être maintenant pris en compte dans l'étude de l'efficacité alimentaire des animaux : le microbiote intestinal, dont on commence à montrer l'implication dans l'efficacité alimentaire, notamment chez les monogastriques (Gabriel et al 2012, Richards et al 2005), et le microbiote ruminal (Morgavi et al 2013).

\section{Conclusion}

Les axes de recherche à développer autour de l'efficacité alimentaire sont multiples et visent surtout à :

- mieux comprendre les processus biologiques sous-jacents aux variations de l'efficacité alimentaire entre les individus en fonction de l'environnement d'élevage (partition des nutriments entre masses lipidiques et protéiques, relation avec le 
niveau d'ingestion, la digestibilité des aliments, la flore intestinale ou ruminale, les fermentations entériques, le comportement alimentaire...) ;

- caractériser phénotypiquement et génétiquement l'efficacité de l'animal dans des systèmes alimentaires différents et à des stades physiologiques différents ;
- étudier la relation entre l'efficacité et les autres performances de l'animal (reproduction, production laitière, santé, qualité de la carcasse et de la viande...) ;

- trouver des indicateurs indirects de la quantité ingérée par l'animal dans le but de pouvoir prédire à moindre coût cette mesure difficile à réaliser à large échelle ;
- développer des équations de prédiction génomiques fiables qui permettraient de sélectionner les animaux sur leur efficacité alimentaire pour des régimes variés.

\section{Références}

Archer J.A., C. Richardson E., Herd R.M. Arthur P.F., 1999. Potential for selection to improve efficiency of feed use in beef cattle: a review. Austr. J. Agricult. Res., 50, 147-161.

Basarab J.A., Price M.A., Aalhus J.L., Okine E.K., Snelling W.M., Lyle K.L., 2003. Residual feed intake and body composition in young growing cattle. Can. J. Anim. Sci., 83, 189-204.

Basso B., Ricard E., Lagüe M., Guy G., MarieEtancelin C., 2010. Mise au point d'un système de mesure individuelle du comportement alimentaire des canards élevés en lot. Journ. Rech. Palmipèdes à Foie Gras, Bordeaux, France, 179-182.

Bastianelli D., Bonnal L., Juin H., MignonGrasteau S., Davrieux F., Carré B, 2010. Prediction of the chemical composition of poultry excreta by near infrared spectroscopy. J. Near Infrared Spectrosc., 18, 69-77.

Berry D.P., Crowley J.J., 2013. Cell biology symposium: Genetics of feed efficiency in dairy and beef cattle. J. Anim. Sci., 91, 1594-1613.

Bewley J.M., Peacock A.M., Lewis O., Boyce R.E., Roberts D.J., Coffey M.P., Kenyon S.J., Schutz M.M., 2008. Potential for estimation of body condition scores in dairy cattle from digital images. J. Dairy Sci., 91, 3439-3453.

Bizeray D., Leterrier C., Constantin P., Picard M., Faure J.M., 2000. Early locomotor behaviour in genetic stocks of chickens with different growth rates. Appl. Anim. Behav. Sci., 68, 231242 .

Bordas A., Tixier-Boichard M., Mérat P., 1992. Direct and correlated responses to divergent selection for residual food intake in Rhode island red laying hens. Br. Poult. Sci., 33,741754.

Boujard T., Dugy X., Genner D., Gosset C., Grig G., 1992. Description of a modular, low cost, eater meter for the study of feeding behavior and food preferences in fish. Physiol. Behav., 52, 1101-1106.

Bouquet A., Fouilloux M.N., Renand G., Phocas F., 2010. Genetic parameters for growth, muscularity, feed efficiency and carcass traits of young beef bulls. Livest. Sci., 129, 3848.

Brun-Lafleur L., Delaby L., Husson F., Faverdin P., 2010. Predicting energy x protein interaction on milk yield and milk composition in dairy cows. J. Dairy Sci., 93, 4128-4143.

C-Lock Inc., 2011. Greenfeed, http://c-lockinc.com/

Conseil National de 1'Alimentation, 2011. Quelle place pour les protéines animales transformées (PAT) dans l'alimentation des porcs, des volailles et des poissons? Avis n ${ }^{\circ} 70 \mathrm{du}$ CNA, adopté le $1^{\mathrm{e}}$ décembre 2011.
CORPEN, 2006. Estimation des rejets d'azote, de phosphore, de potassium, de calcium, de cuivre et de zinc par les élevages avicoles, 55p.

de Verdal H., Mignon-Grasteau S., Bastianelli D., Même N., Le Bihan-Duval E., Narcy A., 2013. Reducing the environmental impact of poultry breeding by genetic selection. J. Anim. Sci., 91, 613-622.

Decruyenaère V., Lecomte P., Demarquilly C., Aufrère J., Dardenne P., Stilmant D., Buldgen A., 2009. Evaluation of green forage intake and digestibility in ruminants using near infrared reflectance spectroscopy (NIRS): Developing a global calibration. Anim. Feed Sci., Technol., $148,138-156$

Dehareng F., Delfosse C., Froidmont E., Soyeurt H., Martin C., Gengler N., Vanlierde A., Dardenne P., 2012. Potential use of milk mid-infrared spectra to predict individual methane emission of dairy cows. Animal, 6, 1694-1701.

Delagarde R., Prache S., D'Hour P., Petit M., 2001. Ingestion de l'herbe par les ruminants au pâturage. Fourrages, 166, 189-212.

Demarquilly C., Chenost M., Giger S., 1995. Pertes fécales et digestibilité des aliments et des rations. In: Nutrition des ruminants domestiques. Ingestion et digestion. Jarrige R., Ruckebush Y., Demarquilly C., Farce M.H., Journet M. (Eds). INRA Editions, 601-647.

Desai A.R, Links M.G., Collins S. A., Mansfield G.S., Drew M.D., Van Kessel A.G., Hill J.E., 2012. Effects of plant-based diets on the distal gut microbiome of rainbow trout (Oncorhynchus mykiss). Aquaculture, 350, 134-142.

de Verdal H., 2011. Intérêt de la sélection en aviculture pour réduire les rejets-Interaction avec l'alimentation. Thèse de l'Université François Rabelais, 326p

Dourmad J.Y., Jondreville C., 2007. Impact of nutrition on nitrogen, phosphorus, $\mathrm{Cu}$ and $\mathrm{Zn}$ in pig manure, and on emissions of ammonia and odours. Livest. Sci., 126, 192-198.

Drouilhet L., Gilbert H., Balmisse E., Ruesche J., Tircazes A., Larzul C., Garreau H., 2013. Genetic parameters for two selection criteria for feed efficiency in rabbits. J. Anim. Sci., 91, 3121-3128.

Dulphy JP., Balch C.C., Doreau M., 1995. Adaptation des espèces domestiques à la digestion des aliments cellulosiques. In: Nutrition des ruminants domestiques. Ingestion et digestion. Jarrige R., Ruckebush Y., Demarquilly C., Farce M.H., Journet M. (Eds). INRA Editions, 759-803.

FAO, 2006. Livestock's Long shadow: environmental issues and options. LEAD, FAO (Eds), 390p.
Gabarrou J.F., Géraert P.A., Picard M., Bordas A., 1997. Diet-induced thermogenesis in cockerels is modulated by genetic selection for high or low residual feed intake. J. Nutr., 127, 2371-2376.

Gabriel I., Konsak B., Mignon-Grasteau S., 2012. Genetic selection of poultry based on digestive capacity - impact on gut microbiota. In: Recent Adv. Anim. Nutr. Garnsworthy P.C., Wiseman J. (Eds). Nottingham University Press, Nottingham, U.K, 197-238.

Gilbert H., Bidanel J.P., Gruand J., Caritez J.C., Billon Y., Guillouet P., Lagant H., Noblet J., Sellier P., 2007. Genetic parameters for residual feed intake in growing pigs, with emphasis on genetic relationships with carcass and meat quality traits. J. Anim. Sci., 85, 3182-3188.

Gill M., Smith P., Wilkinson J.M., 2010. Mitigating climate change: the role of domestic livestock. Animal 4, 323-333.

Glencross B.D., Booth M., Allan G.L., 2007. A feed is only as good as its ingredients - a review of ingredient evaluation strategies for aquaculture feeds. Aqua. Nutr., 13, 17-34.

Griffon M., 2013. Qu'est-ce que l'agriculture écologiquement intensive ? Quae Editions, Paris, France, 224p.

Grima L., 2010. Vers une sélection de l'efficacité d'utilisation de l'aliment chez le poisson, Thèse de doctorat de l'Institut des Sciences et Industries du Vivant et de l'Environnement, Agro Paris Tech, 222p.

Grima L., Quillet E., Boujard T., RobertGranié C., Chatain B., Mambrini M., 2008. Genetic variability in residual feed intake in rainbow trout clones and testing of indirect selection criteria. Genet. Sel. Evol., 40, 607624.

Grima L., Vandeputte M., Ruelle F., Vergnet A., Mambrini M., Chatain B., 2010. In search for criteria to improve residual feed intake in sea bass (Dicentrarchus labrax). Part I: phenotypic relationship between residual feed intake and body weight variations during feed deprivation and re-feeding periods. Aquaculture, 300, 50-58

Halachmi I., Polak P., Roberts D.J., Klopcic M., 2008. Cow body shape and automation of condition scoring. J. Dairy Sci., 91, 4444-4451.

Hegarty R.S., Goopy J.P., Herd R.M., McCorkell B., 2007. Cattle selected for lower residual feed intake have reduced daily methane production. J. Anim. Sci., 85, 1479-1486.

Herd R.M., Arthur P.F., 2009. Physiological basis for residual feed intake, J. Anim. Sci., 87 , E64-E71. doi: 10.2527/jas.2008-1345

Hoch T., Agabriel J., 2004. A mechanistic dynamic model to estimate beef cattle growth 
and body composition: 1. Model description. Agricult. Syst., 81, 1-15.

Idele, 2012. Les coûts de production dans les élevages bovins viande. Enquête annuelle 2011 des réseaux d'élevages. http://idele.fr/domainestechniques/economie-et-gestion-de-lexploitation/couts-de-production/publication/idelesolr/recommends/les-couts-de-productiondans-les-elevages-bovins-viande.htm

Ifip, 2012. Le porc par les chiffres. Ed Ifip, Paris, France, $45 \mathrm{p}$.

Jarret G., Cerisuelo A., Peu P., Martinez J., Dourmad J.Y., 2012. Impact of pig diets with different fibre contents on the composition of excreta and their gaseous emissions and anaerobic digestion. Agricult. Ecosys. Environ., 45, 6204-6209.

Jones F.M., Phillips F.A., Naylor T., Mercer N.B., 2011. Methane emissions from grazing Angus beef cows selected for divergent residual feed intake. Anim. Feed Sci. Technol., 166167, 302-307.

Kause A., Tobin D., Dobly A., Houlihan D., Martin S., Mantysaari E.A., Ritola O. Ruohonen K., 2002. Recording strategies and selection potential of feed intake measured using the X-ray method in Rainbow trout. Genet. Sel. Evol., 38, 389-409.

Kaushik S. J., 1990. Analytical and empirical methods in diet formulation. In: Mediterranean Aquaculture. Floss R., Tort L., Torres P. (Eds). Ellis Horwood, 141-161.

Kelly A.K., McGee M., Crews, D.H., Sweeney Jr. T., Boland T.M., Kenny D.A., 2010 Repeatability of feed efficiency, carcass ultrasound, feeding behavior, and blood metabolic variables in finishing heifers divergently selected for residual feed intake, J. Anim. Sci. $88,3214-3225$

Knap P.W., Su G., 2008. Genotype by environment interaction for litter size in pigs as quantified by reaction norms analysis. Animal, 2, 1742-1747.

Koch R.M., Swiger L.A., Chambers D., Gregory, K.E., 1963. Efficiency of feed use in beef cattle. J. Anim. Sci., 22, 486-494.

Kolstad K., Grisdale-Helland B., Gjerde B., 2004. Family differences in feed efficiency in Atlantic salmon (Salmo salar). Aquaculture, 241, 169-177.

Lawrence P., Kenny D.A., Earley B., Crews D.H., McGee M., 2011. Grass silage intake, rumen and blood variables, ultrasonic and body measurements, feeding behavior, and activity in pregnant beef heifers differing in phenotypic residual feed intake. J. Anim. Sci., 89, 32483261 .

Lawrence P., Kenny D.A., Earley B., McGee M., 2012. Grazed grass herbage intake and performance of beef heifers with predetermined phenotypic residual feed intake classification. Animal 6, 1648-1661.

Le Bail P.Y., Bugeon J., Dameron O., Fatet A., Golik W., Hocquette J.F., Hurtaud C. Hue I., Jondreville C., Joret L., Meunier-Salaun M.C., Vernet J., Nédellec C., Reichstadt M., Chemineau P. ATOL : une ontologie générique pour le phénotypage des animaux d'élevage. In : Phénotypage des animaux d'élevage. Phocas F. (Ed). Dossier, INRA Prod. Anim., 27, 195-208.

Lefaucheur L., Lebret B., Ecolan P., Louveau I., Damon M., Prunier A., Billon Y., Sellier P., Gilbert H., 2011. Muscle characteristics and meat quality traits are affected by divergent selection on residual feed intake in pigs. J. Anim. Sci., 89, 996-1010.

Luiting P., Urff E.M., 1991. Optimization of a model to estimate residual feed consumption in the laying hen. Livest. Prod. Sci., 27, 321-338.

Mambrini M., Médale F., Sanchez M.P., Recalde B., Chevassus B., Labbé L., Quillet E., Boujard T., 2004. Selection for growth in brown trout increases feed intake capacity without affecting maintenance and growth requirements. J. Anim. Sci., 82, 2865-2875.

Martin O., Sauvant D., 2010. A teleonomic model describing performance (body, milk and intake) during growth and over repeated reproductive cycles throughout the lifespan of dairy cattle. 2. Voluntary intake and energy partitioning. Animal, 4, 2048-2056.

Mayes R.W., Lamb C.S., Colgrove P.M., 1986 The use of dosed and herbage n-alkanes as markers for the determination of herbage intake. J. Agricult. Sci., 107, 161-170.

Médale F., Guillaume J.C., 1999. Nutrition énergétique. In : Nutrition et Alimentation des Poissons et Crustacés. Guillaume J., Kaushik S., Bergot P., Métailler R. (Eds). INRA éditions, Paris, France, 87-111.

Meunier-Salaün M.C., Guérin C., Billon Y. Sellier P., Noblet J., Gilbert H., 2013. Divergent selection for residual feed intake in group housed growing pigs: characteristics of physica and behavioural activity according to line and sex. Animal (soumis)

Mignon-Grasteau S., 2007. Prise en compte des interactions entre génotype et environnemen dans les productions avicoles. Journ. Rech. Avicole, Tours, France, 386-393.

Mignon-Grasteau S., Muley N., Bastianelli D. Gomez J., Peron A., Sellier N., Millet N. Besnard J., Hallouis J.M., Carré B., 2004 Heritability of digestibilities and divergent selection for digestion ability in growing chicks fed a wheat diet. Poult. Sci., 83, 860-867.

Mignon-Grasteau S., Bourblanc M., Carré B., Dourmad J.Y., Gilbert H., Juin H., Noblet J. Phocas F., 2010. La réduction des rejets avicoles et porcins par la sélection. INRA Prod. Anim., 23, 415-426.

Montanholi Y.R. Swanson K.C., Schenkel F.S., McBride B.W., Caldwell T.R., Miller S.P. 2009. On the determination of residual feed intake and associations of infrared thermography with efficiency and ultrasound traits in bee bulls. Livest. Sci., 125, 22-30.

Montanholi Y.R., Palme R., Schenkel F.S., McBride B.W., Lu D., Miller S.P., 2010 Assessing feed efficiency in beef steers through feeding behavior, infrared thermography and glucocorticoids. Animal, 4, 692-701.

Moore K.L., Johnston D.J., Graser H.U., Herd R, 2005. Genetic and phenotypic relationships between insulin-like growth factor-I (IGF-I) and net feed intake, fat, and growth traits in Angus beef cattle. Aust. J. Agric. Res., 56, 211 218

Morgavi D.P., Kelly W.J., Janssen P.H., Attwood G.T., 2013. Rumen microbial (meta)genomics and its application to ruminant production. Animal, 7, 184-201.

Muñoz C., Yan T., Wills D.A., Murray S., Gordon A.W., 2012. Comparison of the sulfur hexafluoride tracer and respiration chamber techniques for estimating methane emissions and correction for rectum methane output from dairy cows. J. Dairy Sci., 95, 3139-3148.
Nkrumah J.D., Okine E.K., Mathison G.W., Schmid K., Li C., Basarab J.A., Price M.A. Wang Z., Moore S.S., 2006. Relationships of feedlot feed efficiency, performance and feeding behavior with metabolic rate, methane production and energy partitioning in beef cattle. J. Anim. Sci., 84, 145-153.

Noblet J., Karège C., Dubois S., 1994a. Prise en compte de la variabilité de la composition corporelle pour la prévision du besoin énergétique et de l'efficacité alimentaire chez le porc en croissance. Journ. Rech. Porcine Fr., 26 267-276

Noblet J., Fortune H., Shi X.S., Dubois S., 1994b. Prediction of net energy value of feeds for growing pigs. J. Anim. Sci., 72, 344-354.

Noblet J., Gilbert H., Jaguelin-Peyraud Y., Lebrun T., 2013. Evidence of genetic variability for digestive efficiency in the growing pig fed a fibrous diet. doi: http://dx.doi.org/10.1017/S1751731113000463

Owens F.N., Gill D.R., Secrist D.S., Coleman S.W., 1995. Review of some aspects of growth and development of feedlot cattle. J. Anim. Sci., 73, 3152-3172.

Peyraud J.L., Cellier P., Aarts F., Béline F., Bockstaller C., Bourblanc M., Delaby L., Donnars C., Dourmad J.Y., Dupraz P., Durand P., Faverdin P., Fiorelli J.L., Gaigné C., Girard A., Guillaume F., Kuikman P., Langlais A., Le Goffe P., Le Perchec S., Lescoat P., Morvan T., Nicourt C., Parnaudeau V., Peyraud J.L., Réchauchère O., Rochette P., Vertes F., Veysset P., 2012. Les flux d'azote liés aux élevages, réduire les pertes, rétablir les équilibres. Expertise scientifique collective, rapport, Inra, 503 p.

Phocas F., Bobe J., Bodin L., Charley B., Dourmad J.Y., Friggens N.C., Hocquette J.F., Le bail P.Y., Le Bihan-Duval E., Mormède P., Quéré F., Schelcher F., 2014. Des animaux plus robustes : un enjeu majeur pour le développement durable des productions animales nécessitant 1'essor du phénotypage fin et à haut débit. In : Phénotypage des animaux d'élevage. Phocas F. (Ed). INRA Prod. Anim., 27, 181194.

Pomar C., Pomar J., Dubeau F., Joannopoulos E., Dussault J.P., 2014. The impact of daily multiphase feeding on animal performance, body composition, notrogen and phosphorus excretions, and feed costs in growing-finishing pigs. Animal, in press.

Reverter A., Johnston D.J. Graser H.U., Wolcott M.L., Upton W.H., 2000. Genetic analyses of live-animal ultrasound and abattoir carcass traits in Australian Angus and Hereford cattle. J. Anim. Sci., 78, 1786-1795.

Richards J.D., Gong J., de Lange C. F. M. 2005. The gastrointestinal microbiota and its role in monogastric nutrition and health with an emphasis on pigs: Current understanding, possible modulations, and new technologies for ecological studies. Can. J. Anim. Sci., 85, 421435 .

Richardson E.C., Herd R.M., Archer J.A., Arthur P.F., 2004. Metabolic differences in Angus steers divergently selected for residual feed intake. Aust. J. Exp. Agric., 44, 441-452.

Riffard C., Gallot S., Magdelaine P., 2011. Rapport technique, ITAVI, 57p.

Robelin J., 1986. Bases physiologiques de la production de viande : croissance et développement des bovins. Micol D. (Ed). INRA, Paris, France, 35-60. 
Robinson D.L., 2005. Accounting for bias in regression coefficients with example from feed efficiency. Livest. Prod. Sci., 95, 155-166.

Saintilan R., 2012. Etude de nouvelles approches pour la sélection sur l'efficacité alimentaire chez le porc en croissance et des perspectives de leur mise en place. Thèse de doctorat de l'Institut des Sciences et Industries du Vivant et de 1'Environnement, Agro Paris Tech., 193p.

Saintilan R., Mérour I.,Brossard L., Tribout T., Dourmad J.Y., Sellier P., Bidanel J., van Milgen J., Gilbert H., 2013. Genetics of residual feed intake in growing pigs: Relationships with production traits, J. Anim. Sci., 91, 2542-2554.

Schroder U.J. et Staufenbiel R., 2006. Methods to determine body fat reserves in the dairy cow with special regard to ultrasonographic measurement of backfat thickness. J. Dairy Sci., 89, 1-14.

Silverstein J.T., Hostuttler M., Blemings K.P., 2005. Strain differences in feed efficiency measured as residual feed intake in individually reared Rainbow trout, Onchorhynchus mykiss (Walbaum). Aqua. Res., 36, 704-711.
Thorup V.M, Edwards D., Friggens N.C., 2012. On-farm estimation of energy balance in dairy cows using only frequent body weight measurements and body condition score. J. Dairy Sci., 95, 1784-1793.

van Milgen J., Valancogne A., Dubois S., Dourmad J.Y., Sève B., Noblet J., 2008. InraPorc: A model and decision support tool for the nutrition of growing pigs. Anim. Feed Sci. Technol., 143, 387-405.

\section{Résumé}

Dans un contexte de demande croissante en protéines animales, l'élevage de précision et la sélection animale doivent viser à accroître la compétitivité des systèmes de production et à réduire leur impact environnemental. Les animaux doivent être plus efficaces pour transformer leur ration (réduire l'ingéré tout en maintenant une production stable), en acceptant des ressources alimentaires variées (protéines ou fibres) et en minimisant leurs rejets (totaux ou leurs composantes $\mathbf{N}$ et P, GES). Toutefois, l'efficacité alimentaire est un phénotype complexe qui doit être phénotypé finement pour comprendre les processus biologiques sous-jacents avant d'identifier les leviers d'action pour l'améliorer (efficacité digestive vs métabolique ; efficacité énergétique vs protéique). Ce phénotypage doit être réalisé en conditions variées tant sur le plan du génotype, du niveau alimentaire que de la composition de la ration (teneur en fibres, en acides aminés). Il s'agit aussi d'envisager une approche prédictive de l'ingéré et de l'efficacité alimentaire basée sur des paramètres peu coûteux et faciles à mesurer (croissance, bio-marqueurs...) dans le cadre d'un élevage de précision ou de programmes de sélection génétique, car le phénotypage à large échelle de l'ingéré reste coûteux et parfois difficile à mettre en œuvre. Pour évaluer l'efficience globale des systèmes de productions animales, il s'agit de considérer les réponses des animaux pour des génotypes et des régimes alimentaires contrastés en termes d'efficacité alimentaire et de rejets en quantité et en qualité (GES, N, P), mais aussi de robustesse des animaux (comportement alimentaire, activité générale, santé).

\section{Abstract}

Phenotyping for feed efficiency and its components, a need to improve the efficiency of livestock production

In a context of an increasing demand for animal proteins, precision farming and animal breeding must be goal-oriented to improve production competitiveness and to reduce environmental impact. Animals have to be more efficient in transforming their diet (reducing their feed intake while maintaining production performance), accepting various feeds (protein and fibers) and reducing their wastes (total, N, P, GHG...). However, feed efficiency is a complex phenotype that needs to be deeply phenotyped. This will allow unravelling the biological processes to identify ways to improve feed efficiency (digestive versus metabolic efficiency; energetic versus protein efficiency) according to the genotype, the feed level and the diet composition (fibre content, AA content). Predictive biology of feed intake and feed efficiency is also needed using cost-effective and easy to record traits (growth, biomarkers...) for precision farming and for selective breeding, because large-scale phenotyping for feed intake remains costly. To assess the efficiency of livestock production systems, there is a need to reconsider animal responses according to contrasted genotypes and feeds not only for feed efficiency and wastes in quantity and quality (GHG, N, P), but also for robustness of animals (feeding behavior, general activity, health).

PHOCAS F., AGABRIEL J., DUPONT-NIVET M., GEURDEN I., MÉDALE F., MIGNON-GRASTEAU S., GILBERT H., DOURMAD J.-Y., 2014. Le phénotypage de l'efficacité alimentaire et de ses composantes, une nécessité pour accroître l'efficience des productions animales. In : Phénotypage des animaux d'élevage. Phocas F. (Ed). INRA Prod. Anim., 27, 235-248. 
